\title{
Medical Image of the Week: Yellow Nail Syndrome
}

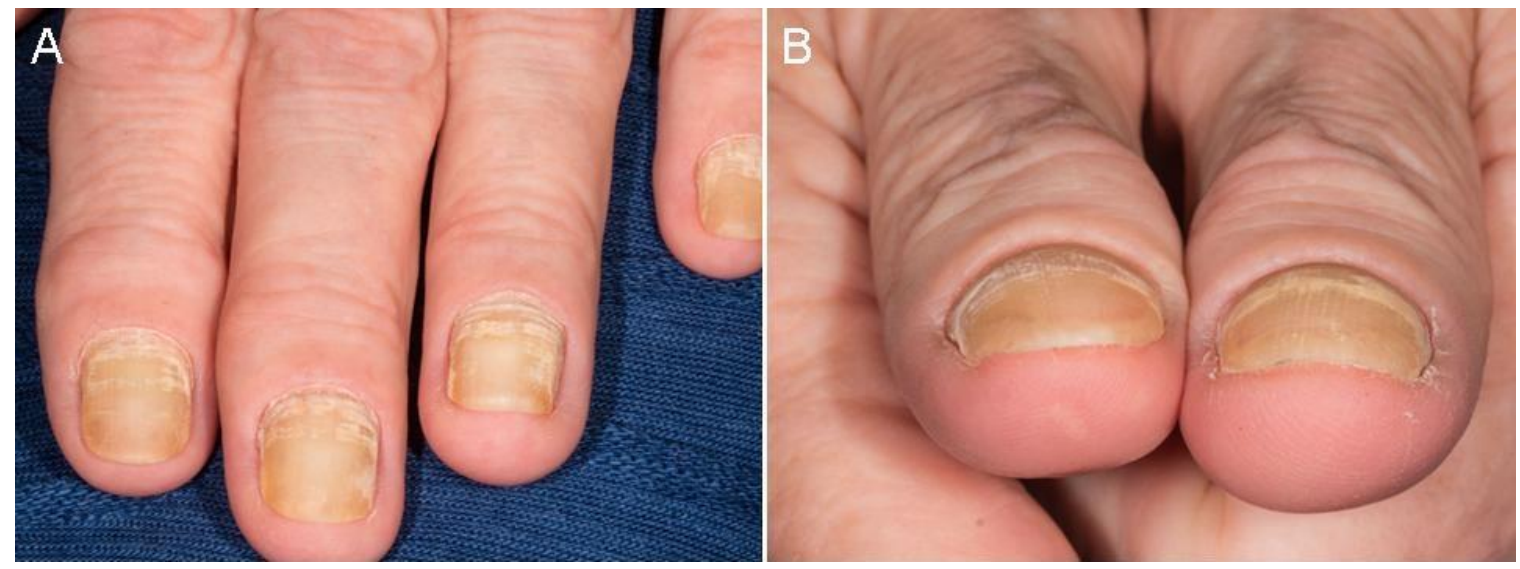

Figure 1. Photographs showing the patient's discolored, yellowish nails.

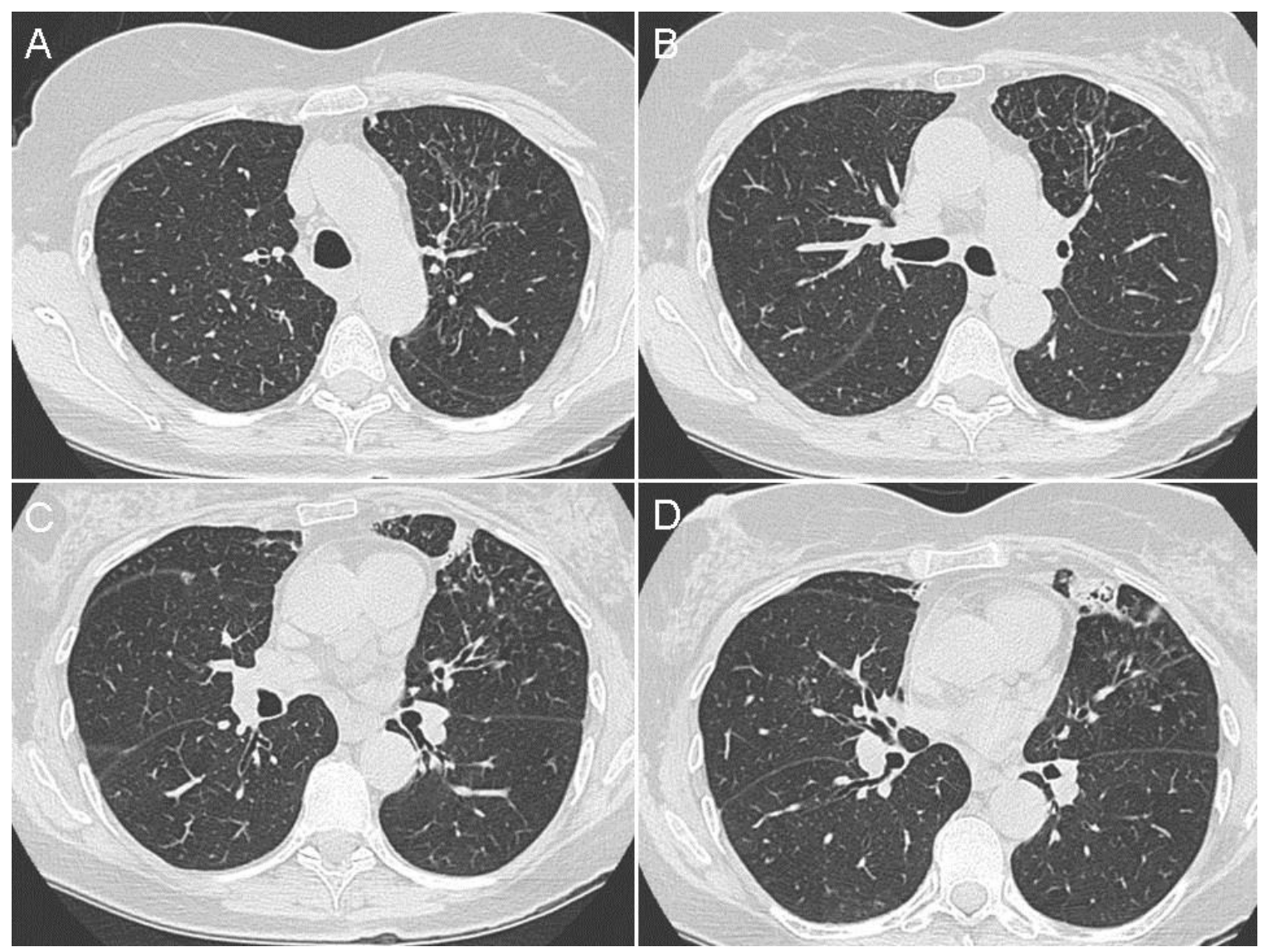

Figure 2. Representative images from the thoracic CT scan in lung windows showing bronchiectasis most marked in the left upper lobe. 
A 67-year-old woman with a previous history of yellow nail syndrome presented with a long history of cough, increased sputum production, recurrent swelling in her lower extremities and recurrent respiratory infections. Physical examination revealed nonpitting edema in the lower extremities and discolored nails (Figure 1). A thoracic CT scan showed bronchiectasis in the left upper lobe (Figure 2). She did not have a history of pleural effusions or chronic sinusitis.

Yellow nail syndrome is very rare disorder associating yellow nail discoloration, bronchiectasis and lymphedema (1). Other frequent manifestations include sinusitis and recurrent pleural effusions. The disease is most frequently isolated but may be associated with other diseases implicating the lymphatic system, autoimmune diseases or cancers. The symptoms result from lymphatic impairment but the cause of the impairment is unknown. Treatment is symptomatic for each component. Vitamin E, combined with fluconazole, is usually prescribed to treat yellow nails and achieves a partial or complete response. Spontaneous resolution is also possible. Although prognosis is usually considered to be good, a Kaplan-Meier survival curve estimated median survival at 132 months, shorter than that of a paired-control population (2).

Lewis J. Wesselius MD

Departments of Pulmonary Medicine

Mayo Clinic Arizona

Scottsdale, AZ USA

\section{References}

1. Vignes S, Baran R. Yellow nail syndrome: a review. Orphanet J Rare Dis. 2017 Feb 27;12(1):42. [CrossRef] [PubMed]

2. Maldonado $\mathrm{F}$, Tazelaar HD, Wang $\mathrm{CW}$, Ryu JH. Yellow nail syndrome: analysis of 41 consecutive patients. Chest. 2008;134:375-81. [CrossRef] [PubMed] 